PROCEEDINGS OF THE

AMERICAN MATHEMATICAL SOCIETY

Volume 133, Number 5, Pages 1463-1467

S 0002-9939(04)07699-3

Article electronically published on December 6, 2004

\title{
A COUNTEREXAMPLE TO THE EXISTENCE OF A LOCAL PLURISUBHARMONIC PEAK FUNCTION AT INFINITY
}

\author{
SUNG-HEE PARK \\ (Communicated by Mei-Chi Shaw)
}

\begin{abstract}
We give an example of an unbounded pseudoconvex Reinhardt domain in $\mathbb{C}^{n}, n \geq 4$, which is Kobayashi complete but admits no local plurisubharmonic peak function at infinity.
\end{abstract}

\section{INTRODUCTION}

Let $G \subset \mathbb{C}^{n}$ be open. We denote by $\mathcal{P S H}(G)$ the set of all plurisubharmonic functions on $G$. For $R>0$ we put $U_{R}(\infty)=U_{R}^{n}(\infty):=\left\{z \in \mathbb{C}^{n}:\|z\|>R\right\}$. We say that a domain $G \subset \mathbb{C}^{n}$ has:

- a local plurisubharmonic peak function $\varphi$ at infinity whenever there exists an $R>0$ such that $\varphi \in \mathcal{C}\left(\bar{G} \cap U_{R}(\infty)\right) \cap \mathcal{P S H}\left(G \cap U_{R}(\infty)\right)$ and

$$
\lim _{G \ni w \rightarrow \infty} \varphi(w)=0>\varphi(z), \quad z \in \bar{G} \cap U_{R}(\infty) ;
$$

- a local plurisubharmonic antipeak function $\varphi$ at infinity whenever there exists an $R>0$ such that $\varphi \in \mathcal{C}\left(\bar{G} \cap U_{R}(\infty)\right) \cap \mathcal{P S H}\left(G \cap U_{R}(\infty)\right)$ and

$$
\lim _{G \ni w \rightarrow \infty} \varphi(w)=-\infty<\varphi(z), \quad z \in \bar{G} \cap U_{R}(\infty) .
$$

These notions were introduced by Gaussier [3]. Recently, some authors showed that the existence of such functions on a given unbounded domain is useful in studying geometric properties of the domain; see e.g. [3], [8], [4], [1]. In particular, the following result can be found in $[3]$.

Theorem 1.1. Suppose that an unbounded domain $G \subset \mathbb{C}^{n}$ admits local plurisubharmonic peak and antipeak functions at infinity. Then $G$ is Kobayashi hyperbolic. If, moreover, every point $p \in \partial G$ has an open neighborhood $U_{p} \subset \mathbb{C}^{n}$ such that any connected component of $G \cap U_{p}$ is taut, then $G$ is taut.

Our aim in this article is to prove that the converses of the statements in Theorem 1.1 may not be true, even though the domain is Kobayashi complete. More precisely, we shall show the following result.

Received by the editors June 3, 2003 and, in revised form, February 1, 2004.

2000 Mathematics Subject Classification. Primary 32T40; Secondary 32F45.

Key words and phrases. Local plurisubharmonic peak function at infinity, local plurisubharmonic antipeak function at infinity.

(C)2004 American Mathematical Society Reverts to public domain 28 years from publication 
Theorem 1.2. For any $n \geq 4$ there exists an unbounded pseudoconvex Reinhardt Hartogs domain in $\mathbb{C}^{n}$ that is Kobayashi complete and does not admit a local plurisubharmonic peak function at infinity.

Finally, in order to understand the (non-)existence of local plurisubharmonic peak and antipeak functions at infinity, we shall shortly give some other examples.

\section{Preliminaries}

Let us recall some basic notation and results that will be needed in the sequel.

By $\|\cdot\|$ we denote the Euclidean norm on $\mathbb{C}^{n},|\cdot|:=\|\cdot\|_{1}$. For $r>0$ we put $E_{r}:=\{\lambda \in \mathbb{C}:|\lambda|<1 / r\}$ and $E:=E_{1}$. Let $u, v: G \rightarrow[-\infty,+\infty)$ be upper semicontinuous with $u+v<0$ on $G$, and set

$$
\Sigma_{u, v}(G):=\left\{(z, w) \in G \times \mathbb{C}^{1}: e^{v(z)}<|w|<e^{-u(z)}\right\} .
$$

Such a domain is called a Hartogs-Laurent domain over $G$. A domain $G \subset \mathbb{C}^{m}$ is called Reinhardt if $\left(\lambda_{1} w_{1}, \cdots, \lambda_{m} w_{m}\right) \in G, \lambda_{1}, \cdots, \lambda_{m} \in \partial E,\left(w_{1}, \cdots, w_{m}\right) \in G$. Obviously, $\Sigma_{u, v}(G)$ is Reinhardt iff $G$ is Reinhardt, $u(z)=u\left(\left|z_{1}\right|, \cdots,\left|z_{n}\right|\right), v(z)=$ $v\left(\left|z_{1}\right|, \cdots,\left|z_{n}\right|\right), z \in G$. Recall that $\Sigma_{u, v}(G)$ is pseudoconvex iff $G$ is pseudoconvex and $u, v \in \mathcal{P S H}(G)$. This property can be found in e.g. [11], [7].

Recall (see e.g. [6], 12]) that the following implications are well known:

Kobayashi complete $\Rightarrow$ taut $\Rightarrow$ Kobayashi hyperbolic $\Rightarrow$ Brody hyperbolic.

In particular, the converse implications are true for any pseudoconvex Reinhardt domain in $\mathbb{C}^{n}$, due to $\mathrm{Fu}[2$ and Zwonek [13].

Let us denote by $\mathcal{S H}(G)$ the family of all subharmonic functions on an open set $G \subset \mathbb{C}$. The following statement is due to Oka [9] (or [11], [5]).

Theorem 2.1. Let $G \subset \mathbb{C}$ be open. Then for any $u \in \mathcal{S H}(G)$ and any continuous curve $\gamma:[0,1] \rightarrow G$ the following is true:

$$
u(\gamma(0))=\limsup _{t \rightarrow 0, t>0} u(\gamma(t)) .
$$

On the other hand, we can easily show the following.

Lemma 2.2. Let $G=\left\{z \in \mathbb{C}^{2}:\left|z_{1} z_{2}\right|<1\right\}$. Then $u \in \mathcal{P S H}(G)$ is bounded from above iff there exists $\tilde{u} \in \mathcal{S H}(E)$ that is bounded from above on $E$ such that $u(z)=\tilde{u}\left(z_{1} z_{2}\right)$ for $z \in G$.

In fact, this auxiliary lemma is a special case of Lemma 4.4.3 in [6].

\section{Proof of Theorem 1.2}

It suffices to find the desired example for $n=4$. For this fix $G:=\left\{z \in \mathbb{C}^{3}\right.$ : $\left.\left|z_{1} z_{2} z_{3}\right|<1\right\}$ and put $u(z):=\max _{j=1,2,3}\left|z_{j}\right|, z \in G$. Clearly, $u \in \mathcal{P S H}(G)$ and $\Sigma:=\Sigma_{u,-\infty}(G)$ is a pseudoconvex Reinhardt Hartogs-Laurent domain in $\mathbb{C}^{4}$. Observe that $\Sigma$ does not contain a non-trivial entire curve (cf. [10]), so it is Kobayashi complete.

Suppose that there exist an $R>0$ and a function $\varphi \in \mathcal{C}(\bar{\Sigma} \cap U) \cap \mathcal{P S H}(\Sigma \cap U)$, where $U:=U_{R}^{4}(\infty)$, such that

$$
\begin{gathered}
\varphi(z, \lambda)<0, \quad(z, \lambda) \in \bar{\Sigma} \cap U, \\
\lim _{\Sigma \ni(z, \lambda) \rightarrow \infty} \varphi(z, \lambda)=0 .
\end{gathered}
$$


Fix a point $a \in \mathbb{C}$ with $|a|=2 R$. Put

$$
\Omega_{a}:=\left\{\left(\left(z_{1}, z_{2}\right), \lambda\right) \in G_{a} \times \mathbb{C}:|\lambda|<\exp \left(-u_{a}\left(z_{1}, z_{2}\right)\right)\right\},
$$

where $G_{a}:=\left\{\left(z_{1}, z_{2}\right) \in \mathbb{C}^{2}:\left|z_{1} z_{2}\right|<1 /(2 R)\right\}$ and $u_{a}:=u(\cdot, a)$ on $G_{a}$. Put $\varphi_{a}\left(z_{1}, z_{2}, \lambda\right):=\varphi\left(z_{1}, z_{2}, a, \lambda\right)$ for $\left(z_{1}, z_{2}, \lambda\right) \in \Omega_{a}$. Observe that

$$
\begin{gathered}
\left\{\left(z_{1}, z_{2}, a, \lambda\right):\left(z_{1}, z_{2}, \lambda\right) \in(\mathbb{C} \times \mathbb{C} \times \mathbb{C} \backslash\{0\}) \cap \Omega_{a}\right\} \subset \Sigma, \\
\varphi_{a}\left(z_{1}, z_{2}, 0\right)=\lim _{\zeta \rightarrow 0, \zeta \neq 0} \varphi\left(z_{1}, z_{2}, a, \zeta\right), \quad\left(z_{1}, z_{2}\right) \in G_{a} .
\end{gathered}
$$

Moreover, $\varphi_{a} \leq 0$ on $\bar{\Omega}_{a}, \varphi_{a} \in \mathcal{P} \mathcal{S H}\left(\Omega_{a}\right)$, and $\varphi_{a}(\cdot, 0) \in \mathcal{P} \mathcal{S H}\left(G_{a}\right)$. By virtue of Lemma 2.2, we may take a function $\tilde{\varphi}_{(a, 0)} \in \mathcal{S H}\left(E_{2 R}\right)$ such that

$$
\varphi_{a}\left(z_{1}, z_{2}, 0\right)=\tilde{\varphi}_{(a, 0)}\left(z_{1} z_{2}\right), \quad\left(z_{1}, z_{2}\right) \in G_{a} .
$$

On the other hand, it follows from Theorem 2.1 that

$$
\tilde{\varphi}_{(a, 0)}(0)=\limsup _{\mathbb{R} \ni t \rightarrow \infty} \tilde{\varphi}_{(a, 0)}\left(\frac{1}{2 R t}\right)=\limsup _{\mathbb{R} \ni t \rightarrow \infty} \varphi_{a}\left(t, \frac{1}{2 R t^{2}}, 0\right)=:-C .
$$

Obviously, $C \geq 0$. If $C=0$, the maximum principle for subharmonic functions implies that $\tilde{\varphi}_{(a, 0)}=0$ on $E_{2 R}$ and so $\varphi(\cdot, a, 0)=0$ on $G_{a}$, a contradiction to our assumption (1). Hence, $C>0$.

In view of (3), we can take a constant $M^{\prime} \gg 1$ so large that

$$
\varphi_{a}\left(t, \frac{1}{2 R t^{2}}, 0\right)<-\frac{3}{4} C, \quad t \in \mathbb{R}, t>M^{\prime} .
$$

Let $t \in \mathbb{R}$ with $t>M^{\prime \prime}:=\max \left\{M^{\prime},|a|\right\}$. Then

$$
\varphi_{a}\left(t, \frac{1}{2 R t^{2}}, 0\right)=\lim _{\lambda \rightarrow 0, \lambda \neq 0} \varphi\left(t, \frac{1}{2 R t^{2}}, a, \lambda\right)<-\frac{3}{4} C .
$$

Hence we can take $M_{t}>t$ so large that

$$
\varphi\left(t, \frac{1}{2 R t^{2}}, a, \lambda\right)<-\frac{1}{2} C, \quad 0<|\lambda|<\exp \left(-M_{t}\right) .
$$

Notice that $u\left(t, 1 /\left(2 R t^{2}\right), a\right)=t$ for any $t \in \mathbb{R}$ with $t>M^{\prime \prime}$ and $\lim _{\mathbb{R} \ni t \rightarrow \infty} M_{t}=\infty$. Therefore, we can take a sequence $\left(t_{j}, 1 /\left(2 R t_{j}^{2}\right), a, \lambda_{j}\right) \in(\mathbb{R} \times \mathbb{R} \times\{a\} \times \mathbb{C}) \cap \Sigma$ such that $t_{j}>M^{\prime \prime}, 0<\left|\lambda_{j}\right|<\exp \left(-M_{t_{j}}\right) \leq \exp \left(-t_{j}\right)$, and $\lim _{j \rightarrow \infty} t_{j}=\infty$. From (4) it follows that

$$
\lim _{j \rightarrow \infty} \varphi\left(t_{j}, \frac{1}{2 R t_{j}^{2}}, a, \lambda_{j}\right) \leq-\frac{1}{4} C<0,
$$

which is a contradiction to our assumption (2). Thus the domain $\Sigma$ has no function $\varphi$ as above, so we are done.

\section{Some eXAmples}

As concrete examples of unbounded domains admitting a local plurisubharmonic peak (or antipeak) function at infinity, it is known that any unbounded domain $G \subset \mathbb{C}^{n}$ with a local holomorphic peak function $f$ at infinity, which means that there is an $R>0$ such that $f$ is holomorphic in $G \cap U_{R}(\infty)$, continuous in $\bar{G} \cap U_{R}(\infty)$, and

$$
\lim _{G \ni w \rightarrow \infty}|f(w)|=1>|f(z)|, \quad z \in \bar{G} \cap U_{R}(\infty),
$$

has also local plurisubharmonic peak and antipeak functions at infinity. Such domains can be found in ([3], Example 3.2.1, Example 3.2.2). 
On the other hand, in the case of non-hyperbolic domains, we have the following simple examples:

(a) A domain with a local plurisubharmonic antipeak function at infinity, and without local plurisubharmonic peak functions at infinity: Let $G^{\prime} \Subset \mathbb{C}^{n}$. Then it is easy to check that any unbounded subdomain $G$ of $G^{\prime} \times \mathbb{C}$ has a local plurisubharmonic antipeak function at infinity. Moreover, according to Theorem 1.1, the domain $G^{\prime} \times \mathbb{C}$ has no local plurisubharmonic peak function at infinity.

(b) A domain without local plurisubharmonic peak and antipeak functions at infinity: Let $h: \mathbb{C}^{n} \rightarrow[0, \infty)$ be upper semicontinuous, $h(\lambda z)=|\lambda| h(z), \lambda \in$ $\mathbb{C}, z \in \mathbb{C}^{n}$, and put $D:=\left\{z \in \mathbb{C}^{n}: h(z)<1\right\}$. Assume that there exists a point $z^{0} \in \mathbb{C}^{n} \backslash\{0\}$ such that $h\left(z^{0}\right)=0$. Let $u: \mathbb{C}^{m} \rightarrow[-\infty, \infty)$ be upper semicontinuous. Consider the following unbounded domain:

$$
\Omega:=\left\{(z, w) \in D \times \mathbb{C}^{m}: h(z) u(w)<1\right\} .
$$

Clearly, it contains a non-trivial entire curve, but has no local plurisubharmonic peak functions at infinity. To check the last assertion, suppose that there exist a constant $R>0$ and a function $\varphi \in \mathcal{C}(\bar{\Omega} \cap U) \cap \mathcal{P S H}(\Omega \cap U)$ with

$$
\lim _{G \ni w \rightarrow \infty} \varphi(w)=0>\varphi(z), \quad z \in \bar{\Omega} \cup U,
$$

where $U:=U_{R}^{n+m}(\infty)$. Fix a point $w^{0} \in \mathbb{C}^{m}$ with $\left\|w^{0}\right\|=2 R$. Since $h=0$ on $\mathbb{C} z^{0}$, one has $\varphi\left(\lambda z^{0}, w^{0}\right)<0, \lambda \in \mathbb{C}$. So, it follows from the Liouville type theorem for subharmonic functions that $\varphi\left(\lambda z^{0}, w^{0}\right) \equiv$ constant $=: 2 C<0$ for any $\lambda \in \mathbb{C}$, so we have $\lim _{|\lambda| \rightarrow \infty} \varphi\left(\lambda z^{0}, w^{0}\right) \leq C<0$, a contradiction to (5).

Therefore, for any $n \geq 3$, the domain $G_{n}:=\left\{z \in \mathbb{C}^{n}:\left|z_{1} \cdots z_{n}\right|<1\right\}$ has no local plurisubharmonic peak function at infinity. Moreover, it has also no local plurisubharmonic antipeak function at infinity. To check this, fix $n \geq 3$ and let $\psi$ be a function defined on $W:=G_{n} \cap U_{R}(\infty)$ for some $R>0$. Suppose that $\psi \in \mathcal{P S H}(W)$ and $\left.\psi\right|_{W}>-\infty=\lim _{W \ni z \rightarrow \infty} \psi(z)$. Fix $a \in \mathbb{C}$ with $|a|=2 R$. Then $\psi(\cdot, a) \in \mathcal{P S H}(\tilde{W})$ where $\tilde{W}:=\left\{\left(z_{1}, \cdots, z_{n-1}\right) \in \mathbb{C}^{n-1}:\left|z_{1} \cdots z_{n-1}\right|<\right.$ $1 /(2 R)\}$, and so $\psi_{a}:=\psi(\cdot, 0, \cdots, 0, a) \in \mathcal{S H}(\mathbb{C})$. Our assumption gives that $\lim _{|\lambda| \rightarrow \infty} \psi_{a}(\lambda)=-\infty$. Hence, the maximum principle for subharmonic functions gives that $\psi_{a} \equiv-\infty$, which is a contradiction to the fact that $\psi_{a}>-\infty$ on $\mathbb{C}$.

Remark 4.1. The previous two examples imply that, in Theorem 1.1, the assumption of the existence of a local plurisubharmonic peak function at infinity cannot be removed.

Remark 4.2. Finally, we would like to mention that it would be interesting to know the existence of a domain that has a local plurisubharmonic peak function at infinity, but does not have local plurisubharmonic antipeak functions at infinity. Moreover, we do not know yet whether a domain that belongs to the same category as the domain obtained in Theorem 1.2 could be found in $\mathbb{C}^{n}, n=2,3$. We should notice that the method used in the proof of this theorem could not be applied to the lower-dimensional cases because of the choice of the domain $G$.

\section{ACKNOWLEDGEMENTS}

The author would like to thank Professor Peter Pflug for his guidance and fruitful discussion. He is also indebted to Professor Marek Jarnicki for his helpful comments. 


\section{REFERENCES}

1. J. Byun, H. Gaussier, and K.-T. Kim, Weak-type normal families of holomorphic mappings in Banach Spaces and characterization of the Hilbert ball by its automorphism group, J. Geom. Anal. 12 (2002), 581-599. MF 1916860 (2003d:58015)

2. S. Fu, On completeness of invariant metrics of Reinhardt domains, Arch. Math. 63 (1994), 166-172. MF 1289299 (95h:32024)

3. H. Gaussier, Tautness and complete hyperbolicity of domains in $\mathbb{C}^{n}$, Proc. Amer. Math. Soc. 127 (1999), 105-116. MF1458872 (99b:32039)

4. H. Gaussier, K.-T. Kim, and S. G. Krantz, A Note on the Wong-Rosay Theorem in Complex Manifolds, Complex Var. Theory Appl. 47 (2002), 761-768. MR1925173|(2003f:32025)

5. P. Jakóbczak and M. Jarnicki, Lectures on holomorphic functions of several complex variables, PS File at http://www.im.uj.edu.pl/ jarnicki/mjp.htm, 2001.

6. M. Jarnicki and P. Pflug, Invariant Distances and Metrics in Complex Analysis, de Gruyter Expositions in Mathematics 9, Walter de Gruyter \& Co., Berlin, 1993. MR1242120 (94k:32039)

7. M. Jarnicki and P. Pflug, Extension of Holomorphic Functions, de Gruyter Expositions in Mathematics 34, Walter de Gruyter \& Co., Berlin, 2000. MR1797263 (2001k:32017)

8. N. Nikolov, Localization of invariant metrics, Arch. Math. 79 (2002), 67-83. MR1923040 (2003f:32014)

9. K. Oka, Sur les fonctions analytiques de plusieurs variables, II Domaines d'holomorphie, J. Sci. Hiroshima Univ., Ser. A 7 (1937), 115-130.

10. S.-H. Park, On hyperbolicity and tautness of certain Hartogs type domains, preprint

11. V. S. Vladimirov, Methods of the Theory of Functions of Many Complex Variables, The M.I.T. Press, 1966. MF 0201669 (34:1551)

12. H. Wu, Normal families of holomorphic mappings, Acta Math. 119 (1967), 193-233. MR:0224869|(37:468)

13. W. Zwonek, On hyperbolicity of pseudoconvex Reinhardt domains, Arch. Math. 72 (1999), 304-314. MR1678013 (99m:32027)

Department of Mathematics, Chonbuk National University, Chonju (Chonbuk), 560756, Republic of KoreA

E-mail address: wshpark@mail.chonbuk.ac.kr 